\title{
10. Zusammenarbeit mit den Mittel- und Osteuropäischen Ländern und den GUS-Staaten
}

\section{OpenEdition}

\section{Journals}

Electronic version

URL: http://journals.openedition.org/sjep/714

DOI: $10.4000 /$ sjep.714

ISSN: 1663-9677

\section{Publisher}

Institut de hautes études internationales et du développement

Printed version

Date of publication: 1 avril 1998

Number of pages: $337-348$

ISSN: $1660-5926$

\section{Electronic reference}

«10. Zusammenarbeit mit den Mittel- und Osteuropäischen Ländern und den GUS-Staaten », Schweizerisches Jahrbuch für Entwicklungspolitik [Online], 17| 1998, Online erschienen am: 29 August 2012, abgerufen am 08 September 2020. URL : http://journals.openedition.org/sjep/714 ; DOI : https://doi.org/10.4000/sjep.714 


\section{ZUSAMMENARBEIT MIT DEN MITTEL- UND OSTEUROPÄISCHEN LÄNDERN UND DEN GUS-STAATEN}

D ie Schweiz hat sich seit 1991 in der Zusammenarbeit mit den Ländern engagiert, deren Wirtschaftsreformen einen Übergang zur Marktwirtschaft gewährleisten. 1,65 Milliarden Franken stehen für die Hilfe an die mittel- und osteuropäischen Länder (MOEL) sowie an die Länder der Gemeinschaft Unabhängiger Staaten (GUS) zur Verfügung. Die Hauptinstrumente der Kooperation sind die technische Zusammenarbeit, die Finanzhilfe und die Kreditgarantien. Die früher auf bestimmte Länder Mitteleuropas konzentrierte Hilfe wird allmählich mehr auf Südosteuropa ausgerichtet. Im Berichtszeitraum haben die eidgenössischen Räte die Beteiligung der Schweiz an der Kapitalerhöhung der Europäischen Bank für Wiederaufbau und Entwicklung (EBWE) gebilligt.

Die Hilfe für die wirtschaftlich am weitesten fortgeschrittenen Länder Mittelund Osteuropas wird vom Entwicklungshilfeausschuss der OECD nicht unter der Rubrik «öffentliche Entwicklungshilfe», sondern in einem getrennten Aggregat «öffentliche Hilfe» aufgeführt. Die Hilfe für die ärmsten Oststaaten wird unter der öffentlichen Entwicklungshilfe verbucht. Tabelle 26 führt die Länder der Region auf, die der Liste der Entwicklungsländer angehören (mit * gekennzeichnet), und die anderen Länder, bei denen die Hilfe im Aggregat «öffentliche Hilfe» enthalten ist.

Die Situation der früheren Ostblockstaaten ist sehr unterschiedlich. Die Wirtschaftsreformen gehen oft mit einer Verschlechterung der sozialen Lage und Arbeitslosigkeit einher und mit der Tatsache, dass die Bevölkerung nicht mehr kostenlos Zugang zu gewissen sozialen und medizinischen Diensten hat. Das Bruttosozialprodukt ist in den mittel- und osteuropäischen Ländern in einer ersten Phase stark gesunken, danach ist es wieder angestiegen. So erreichten einige Transitionsländer (Polen, Slowenien, Tschechien und die Slowakei) 1996 wieder einen Stand ihres BSP, der dem 1989 verzeichneten Niveau entspricht oder nahekommt. Bei anderen Ländern hat sich ein drastischer Rückgang des BSP ergeben: Georgien, wo das BSP 1996 nur noch $18 \%$ des BSP von 1989 erreichte, Aserbeidschan, die Ukraine, Tadschikistan, Armenien, Litauen, Moldawien, Kirgistan und Kasachstan (bei diesen Ländern ist das BSP um mehr als die Hälfte gesunken) ${ }^{1}$. Das BSP der Russischen Föderation erreichte 1996 $56 \%$ seines Stands von 1989. Das BSP pro Kopf ist von einem Land zum anderen sehr unterschiedlich. 1993 war das BSP pro Kopf am höchsten in Slowenien (6490 Dollar) und in Ungarn (3350 Dollar), während es in Rumänien bei 1140 Dollar pro Einwohner und in Albanien bei 340 Dollar lag. Die meisten ausländischen Direktinvestitionen wenden sich auch einer sehr beschränkten Anzahl von Ländern zu. Die kumulativen Investitionen von 1988 bis Mitte 1996 beliefen sich auf 35 Milliarden Dollar, wobei sich aber über $82 \%$ dieser Investitionen auf vier

1. Quelle: «Transition Report update», Europäische Bank für Wiederaufbau und Entwicklung, April 1996. 
Länder konzentrierten: Ungarn (12,1 Milliarden Dollar), Tschechien (6,4 Milliarden Dollar), Russland (6,3 Milliarden) und Polen (3,9 Milliarden) ${ }^{2}$.

\section{$\square$ Rahmenkredite und Organisation der Zusammenarbeit mit den mittel- und osteuropäischen Ländern und der GUS (MOEL/GUS)}

Die rechtliche Grundlage des Programms für die Zusammenarbeit mit den MOEL/GUS ist der 1995 verabschiedete (noch nicht in Kraft getretene) allgemeinverbindliche Bundesbeschluss über die Zusammenarbeit mit den Staaten Osteuropas. Der Beschluss hat eine Laufzeit von zehn Jahren, um den provisorischen Charakter dieser Zusammenarbeit zu betonen. Die Unterstützung des Übergangsprozesses in den MOEL/GUS durch die Schweiz hat hauptsächlich folgende Ziele:

๖ Förderung einer den Grundsätzen der Marktwirtschaft entsprechenden wirtschaftlichen Entwicklung;

๑ Förderung von Rechtsstaatlichkeit, Demokratisierung und Menschenrechten;

๑ Umweltschutz;

- Modernisierung der Infrastruktur (z. B. Energieerzeugung).

Bei schwerwiegenden Menschenrechtsverletzungen oder Diskriminierung von Minderheiten kann der Bundesrat die Zusammenarbeit mit einem Land unterbrechen. Die Konditionalität kann auch wirtschaftlicher Art sein. Die Zusammenarbeit (z.B. gewisse Kofinanzierungen) kann ausgesetzt werden, wenn das Land die von den Bretton-Woods-Institutionen empfohlenen Wirtschaftsreformen nicht einhält.

Die seit 1991 jedes Jahr ausgezahlte Hilfe für die MOEL/GUS beruht auf zwei Rahmenkrediten, die es der Schweiz erlauben, Verpflichtungen für einen Gesamtbetrag von 1,650 Milliarden Franken einzugehen. Der im September 1990 verabschiedete erste Rahmenkredit umfasste einen Betrag von 250 Millionen Franken zugunsten Ungarns, Polens und der früheren Tschechoslowakei. Durch den im Januar 1992 verabschiedeten zweiten Rahmenkredit von 800 Millionen Franken konnte die Hilfe auf Albanien, Bulgarien, die baltischen Staaten, Rumänien und Slowenien erweitert werden. Dieser Rahmenkredit wurde im März 1993 aufgestockt, um die Hilfe auf die Nachfolgestaaten der früheren Sowjetunion (Gemeinschaft Unabhängiger Staaten, GUS) auszudehnen. Bis Ende 1996 beliefen sich die von der Schweiz eingegangenen Verpflichtungen auf 1,55 Milliarden Franken (für beide Rahmenkredite, davon 730 Millionen Franken für Finanzhilfe, 341 Millionen Franken für technische Zusammenarbeit und 480 Millionen Franken für Kreditgarantien). Die humanitäre Hilfe wird durch den Rahmenkredit für die Weiterführung der internationalen humanitären Hilfe finanziert. Die Botschaft des Bundesrates über den dritten Rahmenkredit ist in Vorbereitung und dürfte den eidgenössischen Räten im Herbst 1998 unterbreitet werden.

Die früher der Politischen Abteilung I des EDA zugeordnete Abteilung für die Zusammenarbeit mit Osteuropa wurde 1996 der DEZA eingegliedert. Die Programme für technische Zusammenarbeit, die humanitäre Hilfe und die allge-

2. Quellen: EBWE, EWG/UNO, Schätzungen des BAWI, vom Magazin «Die Volkswirtschaft» 2/1997 übernommen. 
meine Koordination der Hilfe wurden der DEZA übertragen, während das BAWI für die finanzielle Zusammenarbeit (Finanzhilfe, Kreditgarantien, Zahlungsbilanzhilfe) und die Handels- und Investitionsförderung zuständig ist.

\section{Auszahlungen 1996}

1996 belief sich die Hilfe für die MOEL/GUS auf 143,3 Millionen Franken (plus 27,6 Millionen Franken für humanitäre Hilfe), davon 67,2 Millionen Franken für Finanzhilfe, 58,7 Millionen für technische Zusammenarbeit, 14 Millionen Franken für Handels- und Investitionsförderung, wobei die Zahlungsbilanzhilfe 3,4 Millionen Franken betrug (Kreditgarantien geben zu keinen Auszahlungen Anlass).

Von den 143,3 Millionen Franken für technische Zusammenarbeit und Finanzhilfe wurden 42,1 Millionen Franken an Länder ausgezahlt, die der Liste der Entwicklungsländer angehören (dieser Betrag ist somit in der öffentlichen Entwicklungshilfe (APD) eingeschlossen), während 101,2 Millionen Franken an die Transitionsländer gingen (dieser Betrag ist in einem Aggregat öffentlicher Hilfe für Transitionsländer berücksichtigt). Die Hilfe der Schweiz für die Transitionsländer machte in den letzten Jahren 0,05\% des BSP aus, während sich das gewogene Mittel für die Gesamtheit der DAC-Länder auf 0,04\% des BSP belief.

\section{$\square$ Instrumente der Zusammenarbeit}

Die Hauptinstrumente der Kooperation mit den osteuropäischen Ländern umfassen die technische Zusammenarbeit und die finanzielle Zusammenarbeit (Finanzhilfe, Kreditgarantie, Zahlungsbilanzhilfe, Handels- und Investitionsförderung). Durch die finanzielle Hilfe lassen sich für die Partnerländer vordringliche Projekte finanzieren, die nicht durch die Privatwirtschaft finanzierbar sind und für welche die schweizerische Wirtschaft Ausrüstungsgüter oder Dienstleistungen bereitstellen kann. Die Aufteilung der Zusammenarbeit unter den verschiedenen Instrumenten und die Arbeitsteilung zwischen den beiden betroffenen Ämtern (DEZA und BAWI) gehen nicht ohne Probleme vor sich und schliessen insbesondere die Gefahr allfälliger Doppelspurigkeiten mit ein. Die technische Zusammenarbeit und die Finanzhilfe sind oft nicht voneinander zu trennen. Gleichwohl ist die DEZA für die technische Zusammenarbeit und das BAWI für die Finanzhilfe zuständig ${ }^{3}$. Deshalb hat der Bundesrat im November 1997 beschlossen, die Koordination zwischen beiden Bundesämtern klar zu verstärken und die Aufgabenteilung zu klären (Übertragung des Gesundheitssektors an die DEZA, Übertragung des Bankensektors an das BAWI). Ein weiteres Problem liegt darin, dass ein bedeutender Anteil des Rahmenkredits für die Kreditgarantien bereitgestellt wird. Einige würden es vorziehen, dass der Anteil der Mittel, die für Projekte technischer Zusammenarbeit ausgezahlt werden können, im nächsten Rahmenkredit erhöht wird. Die Arbeitsgemeinschaft der Hilfswerke ist ihrerseits der Meinung, dass die Zusammenarbeit mit den MOEL/GUS zu sehr auf die Interessen der Schweizer Exporteure ausgerichtet ist.

3. Für mehr Informationen betreffend die Probleme der Koordination der Hilfe verweisen wir auf das Dossier über die Zusammenarbeit mit den Oststaaten in der Zeitschrift Mosquito, Nr. 2, April 1997. 


\section{- Technische Zusammenarbeit}

Diese Zusammenarbeit hat zum Ziel, Fähigkeiten in den Transitionsländern zu entwickeln. Man stellt dem Partnerland im allgemeinen qualifizierte Experten für Beratung und Ausbildung, für die Planung und Durchführung bestimmter Arbeiten zur Verfügung ${ }^{4}$.

\section{Finanzhilfe}

Die Partnerländer können dem BAWI Projektvorschläge in den prioritären Bereichen und Sektoren unterbreiten, die in den Finanzhilfeabkommen festgelegt sind, welche mit den meisten Transitionsländern abgeschlossen werden. Das BAWI prüft dann mit der Hilfe externer Konsulenten die finanzielle, technische und institutionelle Machbarkeit der Projekte sowie ihre Umweltauswirkungen. Die Unterstützung erfolgt mittels bilateraler Projekte oder durch Kofinanzierungen mit internationalen Institutionen wie der Weltbank oder der Europäischen Bank für Wiederaufbau und Entwicklung (EBWE). Die Finanzhilfe wird in Form von nicht rückzahlbaren Finanzierungszuschüssen für Projekte gewährt, die nicht rentabel genug wären, um durch eine kommerzielle Finanzierung unterstützt werden zu können.

Die wirtschaftlichen Rückflüsse der Finanzhilfe für die MOEL/GUS, in Form von Aufträgen, können für manche schweizerische Unternehmen bedeutend sein. Diese Aufträge können durch den Zugang zu Märkten und durch weitere künftige Aufträge mittelfristig interessante kommerzielle Aussichten eröffnen. In einigen Fällen können Spannungen zwischen Schweizer Firmen und dem BAWI auftreten, wenn die Interessen der Exportunternehmen nicht mit den wirklichen Entwicklungsbedürfnissen der Partnerländer übereinstimmen, während das letztgenannte Kriterium vorherrschend bleiben sollte 5 . Das BAWI muss auch darüber wachen, dass die Angebote der Schweizer Unternehmen konkurrenzfähig sind.

\section{Exportkreditgarantien}

Diese Kreditgarantien werden zur Deckung des Risikos eingesetzt, das mit der Finanzierung von Ausfuhren von Gütern und Dienstleistungen an Unternehmen oder für Infrastrukturprojekte verbunden ist, wobei man bei diesen Lieferungen einen genügenden Ertrag für die Rückzahlung und Verzinsung erwartet. Solche Garantien werden für Länder vergeben, bei denen die Exportrisikogarantie (ERG) wegen eines als zu hoch erachteten Risikos nicht (oder nur teilweise) verfügbar ist. Für diese Verpflichtungen wurden bedeutende Mittel aus den ersten beiden Rahmenkrediten, nämlich 480 Millionen Franken, bis Ende 1996 mobilisiert (dabei handelt es sich selbstverständlich um Verpflichtungen ohne effektive Auszahlungen). Hierdurch konnten Schweizer Exporteuren Garantien in den folgenden wichtigsten Ländern gewährt werden: Polen (116 Millionen Franken), Russland (100 Millionen Franken), Usbekistan/Kasachstan (50), Bulgarien (45), Kroatien (35) und der Ukraine (35). Die Kreditgarantien sind nur im Schadenfall einforderbar.

4. Die DEZA-Abteilung für die Zusammenarbeit mit Osteuropa und der GUS gibt eine vollständige Liste aller laufenden oder abgeschlossenen Projekte der technischen Zusammenarbeit nach Empfängerländern heraus. DEZA, Eine Dokumentation über die Projekte der technischen Zusammenarbeit mit den osteuropäischen Staaten, 2. Rahmenkredit, Bern, DEZA, Januar 1998 (periodisch aktualisierte Liste).

5. Schweizerischer Bankverein, Der Monat in Wirtschaft und Finanz, 1. Februar 1997 


\section{Zahlungsbilanzhilfe}

Die Zahlungsbilanzhilfen erlauben es den Partnerländern, dringend benötigte Exportgüter zu kaufen oder ihre Währungsreserven zu erhöhen. Die Darlehen werden von der Nationalbank vergeben und vom Bund garantiert. In einigen Fällen kann die Hilfe in Geschenkform (unter dem Rahmenkredit für Zusammenarbeit) gewährt werden. 1995 und 1996 hat die Schweiz Zahlungsbilanzhilfen für Albanien und für Mazedonien in Höhe von jeweils 6 und 7 Millionen Franken gewährt.

\section{口 Entschuldung}

Die Schweiz hat an internationalen Entschuldungsaktionen für Polen (1993) und Bulgarien (1995) teilgenommen. Diese Aktionen erfolgten in Form von Debt for Nature Swaps, wobei die Empfängerländer die sich aus dem Schuldenerlass ergebenden Gelder zur Finanzierung von Projekten im Umweltbereich verwendeten.

\section{Handels- und Investitionsförderung}

Das BAWI unterstützt Projekte mit dem Ziel, die Ausfuhren der osteuropäischen Staaten zu stärken und ihre Integration in den Welthandel zu erleichtern, namentlich durch Ausbildungsmassnahmen. Ein Teil der Hilfe erfolgt über schweizerische Organisationen, wie zum Beispiel die Schweizerische Zentrale für Handelsförderung (OSEC) und die Stiftung SOFI (Swiss Organisation for Facilitation Investments). Der andere Teil der Hilfe läuft über internationale Organisationen, wie das Internationale Handelszentrum (ITC), die UNCTAD, die WTO, die EFTA oder die Institutionen der Weltbankgruppe. Der Auftrag für die Durchführung des Programms zur Geschäftsanbahnung zwischen schweizerischen Unternehmen und dem Privatsektor der Entwicklungs- und Transitionsländer wurde nach einer Ausschreibung der Revisions- und Unternehmensberatungsfirma KPMG Fides übertragen. Diese hat im Juli 1997, zusammen mit dem BAWI, die Stiftung SOFI mit dem Ziel gegründet, die schweizerischen Investitionen in fünfzehn Entwicklungsländern und neun Transitionsstaaten aktiv zu fördern. Die Stiftung soll es erlauben, die Investitionen zu steigern und den Know-How-Transfer zwischen Schweizer Firmen und Unternehmen der Partnerländer zu fördern.

\section{$\checkmark$ Humanitäre Hilfe}

Die humanitäre Hilfe für Osteuropa hat seit 1991, vor allem wegen des Konflikts im früheren Jugoslawien, massiv zugenommen. Die humanitäre Hilfe war von 1991 bis 1992 von 8 auf 48 Millionen Franken angestiegen und lag zwischen 1993 und 1995 bei 40 bis 45 Millionen Franken. 1996 belief sich die humanitäre Hilfe auf 27,6 Millionen Franken, davon 16,9 Millionen für die Opfer des Konflikts in Ex-Jugoslawien, 4,4 Millionen für die Kaukasusrepubliken und 4,1 Millionen Franken in Russland.

Betreffend die jüngste Entwicklung der humanitären Hilfe und die neue Botschaft des Bundesrates, insbesondere den Teil über die Hilfe für Bosnien, siehe das Kapitel über Entwicklungszusammenarbeit und humanitäre Hilfe im Jahrbuch 1998, S. 323. 


\section{口 Multilaterale Hilfe}

Bei der Finanzierung des Transitionsprozesses in den MOEL/GUS sind folgende internationale Finanzinstitutionen tätig (nach der Grössenordnung ihres Beitrags aufgeführt): Europäische Bank für Wiederaufbau und Entwicklung (siehe unten), IWF und Weltbank, Internationale Finanzgesellschaft, Europäische Investitionsbank, und in geringerem Masse die Asiatische Entwicklungsbank (Kasachstan, Kirgistan und Usbekistan sind Mitglieder der ADB). Weitere in den MOEL/GUS tätige Institutionen, denen die Schweiz als Mitglied angehört, sind: Europarat, EFTA, OSZE, WTO und OECD.

\section{$\square$ Kapitalerhöhung der Europäischen Bank für Wiederaufbau und Entwicklung (EBWE)}

Der Bundesrat hat im November 1996 seine Botschaft über die Beteiligung der Schweiz an der Kapitalerhöhung der Europäischen Bank für Wiederaufbau und Entwicklung (EBWE) vorgelegt. Gemäss den Statuten darf der Betrag der Darlehensvergabe, der Beteiligungen und Garantien der Bank den Gesamtbetrag ihres Grundkapitals nicht überschreiten. Im April 1996 haben die Gouverneure der EBWE beschlossen, das Kapital der Bank durch eine Erhöhung von 10 auf 20 Milliarden ECU zu verdoppeln. Die Mitgliedsländer wurden aufgefordert, neue Aktien für einen dem Anfangskapital entsprechenden Anteil zu zeichnen, was bei der Schweiz einem Anteil von 2,28\% des Kapitals entspricht. Die Beteiligung der Schweiz an der Kapitalaufstockung entspricht einer Verpflichtung von 228 Millionen ECU (was bei einem Umrechnungskurs von 1,50 Franken für 1 ECU 342 Millionen Franken ausmacht). Von diesem Betrag sind lediglich 22,5\%, das heisst 77 Millionen Franken, effektiv auszuzahlen. Der Restbetrag von 265 Millionen Franken stellt Garantiekapital dar.

Die Schweiz ist mit einem Exekutivdirektor im 23 Mitglieder zählenden Verwaltungsrat der EBWE vertreten. Der schweizerische Exekutivdirektor steht, ähnlich wie bei der Weltbank ${ }^{6}$, an der Spitze einer Stimmrechtsgruppe, welche folgende Länder umfasst: Aserbeidschan, Kirgistan, Liechtenstein, Türkei, Turkmenistan und Usbekistan. Dem Verwaltungsrat obliegt die Leitung der Bank (Annahme der Projekte sowie der Länder- und Sektorstrategien). Das oberste Entscheidungsgremium ist der Gouverneursrat, der normalerweise einmal im Jahr zur Jahresversammlung zusammentritt. Die Gouverneure, unter ihnen JeanPascal Delamuraz für die Schweiz, prüfen, ob das Mandat der Bank eingehalten wird, setzen die operationellen Prioritäten fest, nehmen neue Mitglieder auf und wählen den Präsidenten.

\section{AKTIVITÄTEN DER EUROPÄISCHEN BANK FÜR WIEDERAUFBAU UND ENTWICKLUNG}

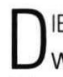
E EBWE wurde 1990 auf Initiative François Mitterands gegründet, um für die aus den politischen und wirtschaftlichen Umwälzungen im Osten hervorgegangenen Länder koordinierte Hilfe bereitzustellen. Die Aufgabe der Bank besteht darin, den Übergang dieser Länder zur Marktwirtschaft zu fördern und ihre Integration in die Weltwirtschaft zu erleichtern. Die Investitionen der EBWE kommen 26 Ländern zugute, und die Bank zählt 60 Mitglieder (58 Länder, darunter die Mitglieder der Europäischen Union und andere europäische Länder, Transitionsländer, die Vereinigten Staaten, Japan und Kanada sowie die Europäische Kommission und die Europäische Investitionsbank).

6. Die für die Bretton-Woods-Institutionen eingesetzte Gruppe umfasst folgende Länder: Aserbeidschan, Kirgistan, Usbekistan, Polen, Tadschikistan, Turkmenistan. 
Die Finanzierungsinstrumente der Bank sind die Darlehen, Kapitalbeteiligungen und Bürgschaften. Mindestens $60 \%$ der Anlagemittel müssen dem privaten Sektor zukommen. Die Aktivitäten im Bereich der technischen Zusammenarbeit dienen dazu, die von der EBWE finanzierten Projekte zu identifizieren, vorzubereiten und zu verwalten, wie auch Dienstleistungen, Beratung und Ausbildung zu finanzieren. So erlaubten es die von der EBWE finanzierten Projekte beispielsweise, die Privatisierung von Banken zu unterstützen und Basisinfrastrukturen zu entwickeln und zu modernisieren.

Die von der EBWE bewilligten kumulativen Finanzierungen beliefen sich bis Ende 1995 auf 7,85 Milliarden ECU (1 ECU = 1,50 Franken). Die wichtigsten Empfängerländer waren Russland (1,7 Milliarden ECU), Ungarn (1,1 Milliarden), Polen, Rumänien, die tschechische und die slowakische Republik, Slowenien und die Ukraine (diese acht Länder machen $72 \%$ der von der EBWE zugesagten Gesamtfinanzierungen aus). Auf der Jahresversammlung der EBWE Anfang April 1997 haben sich mehrere Länder, darunter die Vereinigten Staaten und die Schweiz, für einen allmählichen Rückzug der EBWE aus den wirtschaftlich am weitesten fortgeschrittenen Transitionsländern ausgesprochen, um die Anstrengungen auf jene Länder zu konzentrieren, die sich in einem noch weniger fortgeschrittenen Stadium befinden. Dieses Graduierungsprinzip wurde angenommen, auch wenn einige europäische Staaten und die betroffenen Länder ihre Bedenken zum Ausdruck brachten.

Die Sektoren, welche am meisten Finanzierungen erhielten, sind folgende: Finanzen und Dienstleistungen an Unternehmen (2,7 Milliarden ECU bis Ende 1995), Transport (1,3 Milliarden ECU), Telekommunikationen, Fertigungsindustrie, Energie und Montanindustrie (diese Bereiche machen zusammen $92 \%$ der gesamten Projekte der EBWE aus).

Die Bank kann eine Katalysatorrolle spielen, indem sie externe Finanzierungen für ihre Projekte durch Kofinanzierungen mit privaten Partnern, Banken, Exportkreditagenturen und Regierungen mobilisiert. Die Wahl der Projekte orientiert sich an mehreren Prioritäten: Förderung des Privatsektors, insbesondere der KMU, Unterstützung der Finanzintermediäre (lokale Banken), Erhöhung der Beteiligungen an den lokalen Unternehmen und Banken. Die Gründungsakte der Bank setzt das Prinzip der Förderung einer nachhaltigen und ökologischen Entwicklung fest.

Quelle: Botschaft über die Beteiligung der Schweiz an der Kapitalerhöhung der Europäischen Bank für Wiederaufbau und Entwicklung (EBWE), vom 13. November 1996, Botschaft Nr. 96.089 .

Die im Rahmen der Projekte vergebenen Warenlieferungs- und Dienstleistungsaufträge sind Gegenstand internationaler Ausschreibungen. Die Botschaft hebt hervor, dass die wirtschaftlichen Rückflüsse an die schweizerische Wirtschaft interessant sein können, insbesondere für die Bereiche Energie, Maschinenbau, Fernmeldewesen, Agro- und Textilindustrie. Die Schweiz finanziert durch den Rahmenkredit über die Zusammenarbeit mit Osteuropa (BAWI) einen Treuhandfonds (Trust Fund), der es der EBWE erlaubt, schweizerische oder ausländische Konsulentenfirmen mit der Vorbereitung und Begleitung von Investitionsprojekten zu beauftragen (vor allem im Energiebereich, namentlich mit der Studie über die Wiederinstandsetzung des gesamten Energiesektors in Bosnien). Die Schweiz kofinanziert aus den Finanzhilfefonds des BAWI Projekte der EBWE. Die Unternehmen können im Rahmen der von der EBWE finanzierten Projekte Lieferungen tätigen und diese Beteiligung kann zur Öffnung neuer Märkte für die Schweizer Unternehmen beitragen. Die Schweiz hat mit 5 Millionen Franken zum Spezialfonds für Kleinunternehmen in Russland (Kredite für Kleinbetriebe) beigetragen. Durch die Risikokapitalfonds können Darlehen für die Gründung oder Entwicklung bestimmter Unternehmen gewährt werden.

Die Schweiz leistet auch ihren Beitrag zum nuklearen Sicherheitskonto. Mehrere Kernkraftwerke haben eine veraltete Ausrüstung und werden als sehr gefährlich eingestuft. Jedoch ist die Stillegung bestimmter Kraftwerke in manchen Fällen mangels einer Ersatzenergie nicht in Betracht zu ziehen. Die EBWE interveniert 
bei der Lieferung von Sicherheitsausrüstungen. Die Schweiz hat 20 Millionen Franken zu diesem Nuklearfonds beigetragen. Die Ukraine hat die internationale Gemeinschaft und die EBWE um Hilfe ersucht, um die Verstärkung des Schutzsarkophags um das Kernkraftwerk von Tschernobyl zu finanzieren, aber auch für den Ausbau von zwei Atomkraftwerken, die es gestatten, die anderen Reaktoren von Tschernobyl längerfristig zu ersetzen. Die sieben grössten Industriestaaten (G-7), die Europäische Union und andere Geberländer verpflichten sich, finanzielle Beiträge für die Renovierung der Kernkraftwerke und die Verstärkung des Sarkophags zu leisten (der Beitrag der Schweiz beläuft sich auf 6,4 Millionen Franken $)^{7}$.

Der Nationalrat hat die Beteiligung der Schweiz an der Kapitalerhöhung der Europäischen Bank für Wiederaufbau und Entwicklung am 29. April 1997 mit 161 Ja-Stimmen, 7 Nein-Stimmen und 5 Enthaltungen angenommen. Nationalrat Hans Steffen (SD, ZH) wollte in den Bundesbeschluss einen Artikel aufnehmen, der besagte, dass sich die Schweiz an einer allfälligen späteren Kapitalerhöhung nicht mehr beteiligen werde. Dieser Vorschlag wurde mit $153 \mathrm{zu} 19$ Stimmen abgelehnt. Der Ständerat hat den Bundesbeschluss am 17. Juni 1997 ohne Gegenstimmen angenommen.

\section{$\square$ Geographische Aufteilung der Zusammenarbeit mit den Ländern Mittel- und Osteuropas und den GUS-Staaten}

Unter Berücksichtigung sämtlicher Verpflichtungen der Schweiz von 1990 bis Ende 1996 und sämtlicher Instrumente der Zusammenarbeit waren die Hauptempfängerländer der schweizerischen öffentlichen Hilfe (in abnehmender Grössenordnung der Verpflichtungen aus dem ersten und zweiten Rahmenkredit): Polen, die Russische Föderation, Bulgarien, Albanien, Rumänien und Ungarn. Die Zusammenarbeit mit den MOEL/GUS hatte sich zu Anfang eher auf die mitteleuropäischen Länder konzentriert. Im September 1995 leitete die DEZA eine Strategie der Konzentration der Hilfe auf die südosteuropäischen Länder (Albanien, Bulgarien, Mazedonien und Rumänien) und bestimmte GUS-Staaten (Russland, Ukraine und Kirgistan) ein. Grosse Bedeutung wird auch dem Wiederaufbauprogramm in Bosnien beigemessen. Als Folge der Entscheidung, die Hilfe auf die südosteuropäischen Staaten zu konzentrieren, wurde das Koordinationsbüro in Warschau Ende 1997 geschlossen. Polen ist seit 1990 das Hauptempfängerland der schweizerischen Zusammenarbeit gewesen. Die DEZA hat seit diesem Zeitpunkt Projekte in Höhe von rund 40 Millionen Franken finanziert. Das BAWI hat Finanzbeiträge von rund 115 Millionen Franken (vor allem im Gesundheits- und Umweltbereich) geleistet. Die Kreditgarantien beliefen sich auf 116 Millionen Franken. Die letzten Projekte werden bis Ende 1999 abgeschlossen sein. Die Zusammenarbeit mit Ungarn wird auch zu diesem Zeitpunkt zu Ende gehen, später auch die Zusammenarbeit mit der Slowakei. Die Kooperation mit den baltischen Staaten, der Tschechischen Republik und Slowenien hat bereits 1997 ein Ende genommen. 
Tabelle Nr. 26

\section{Zusammenarbeit mit den Ländern Mittel- und Osteuropas und den GUS-Staaten}

\begin{tabular}{|c|c|c|c|c|}
\hline $\begin{array}{r}\text { Tech } \\
\text { Zusammen }\end{array}$ & $\begin{array}{l}\text { ische } \\
\text { rbeit }\end{array}$ & Finanzhilfe & $\begin{array}{r}\text { Humanitäre } \\
\text { Hilfe }\end{array}$ & Insgesamt \\
\hline Mitteleuropa & 22.7 & 19.2 & - & 41.9 \\
\hline Polen & 3.1 & 13.2 & & 16.3 \\
\hline Ungarn & 6.0 & 0.1 & & 6.1 \\
\hline Tschechien & 1.4 & 2.8 & & 4.2 \\
\hline Slowakei & 1.4 & - & & 1.4 \\
\hline Slowenien $^{1}$ & 0.9 & - & & 0.9 \\
\hline Litauen & 0.5 & - & & 0.5 \\
\hline Lettland & 0.4 & - & & 0.4 \\
\hline Regionale Projekte & 9.0 & 3.1 & & 12.1 \\
\hline Südosteuropa & 23.0 & 29.8 & 16.9 & 69.7 \\
\hline Bosnien-Herzegowina $^{1}$ & 7.0 & 11.4 & - & 18.4 \\
\hline Verschiedene Staaten Ex-Jugos. ${ }^{1}$ & - & - & 16.9 & 16.9 \\
\hline Albanien $^{1}$ & 4.9 & 10.0 & 0.0 & 15.0 \\
\hline Rumänien & 4.9 & 0.1 & - & 5.0 \\
\hline Bulgarien & 3.8 & 0.0 & - & 3.8 \\
\hline Mazedonien $^{1}$ & 0.2 & 3.4 & 一 & 3.6 \\
\hline Regionale Projekte & 2.1 & 4.9 & - & 7.0 \\
\hline GUS & 13.0 & 35.6 & 10.6 & 59.2 \\
\hline Russland & 5.6 & 15.4 & 4.1 & 25.1 \\
\hline Weissrussland & - & 6.0 & 0.2 & 6.2 \\
\hline Ukraine & 0.5 & 3.1 & 一 & 3.6 \\
\hline Kirgistan $^{1}$ & 2.1 & 1.3 & - & 3.4 \\
\hline Moldau $^{2}$ & - & 2.5 & 一 & 2.5 \\
\hline Tadschikistan $^{1}$ & - & - & 1.8 & 1.8 \\
\hline Georgien $^{1}$ & - & 0.9 & - & 0.9 \\
\hline Usbekistan $^{1}$ & - & - & 0.2 & 0.2 \\
\hline Regionale Projekte & 4.7 & 6.4 & 4.4 & 15.6 \\
\hline Gesamtbetrag & 58.7 & 84.6 & 27.6 & 170.8 \\
\hline
\end{tabular}

1. Diese Länder gehören der Liste der Entwicklungsländer an.

2. Die Republik Moldau wird seit Anfang 1997 in der Liste der Entwicklungsländer aufgeführt.

Quelle: Jahresbericht über die internationale Zusammenarbeit der Schweiz 1996, Bern, DEZA und BAWI, 1997. 


\section{Kirgistan}

Seit 1994 hat die Schweiz ein provisorisches Verbindungsbüro von Intercooperation in Bischkek finanziell unterstützt, um das Schweizer Kooperationsprogramm (DEZA und BAWI) in Kirgistan zu koordinieren, wie auch für die Projekte der vor Ort tätigen NRO (Intercooperation, Caritas und Helvetas). 1997 wurde dieses Büro in ein offizielles Koordinationsbüro umgewandelt. Das Kooperationsprogramm in Kirgistan wurde anlässlich der Prüfung der schweizerischen Politik der Entwicklungszusammenarbeit durch den Entwicklungshilfeausschuss der OECD von einer Delegation des DAC besichtigt ${ }^{8}$. Kirgistan ist das erste Partnerland der Schweiz unter den GUS-Staaten Zentralasiens. Die Schweiz kofinanziert ein Weltbank-Projekt zur Entwicklung wirtschaftlich lebensfähiger Finanzmärkte im ländlichen Bereich (nach dem Prinzip der Raiffeisenkassen). Sie unterstützt ferner ein UNDP-Programm zur Förderung von Demokratie und Menschenrechten. Der Bund finanziert Kooperationsprogramme schweizerischer NRO: Regieprojekte zur Verarbeitung von Milchprodukten und zur Entwicklung im Forstsektor (Intercooperation); Projekt zur Unterstützung der Privatinitiative in der Landwirtschaft (landwirtschaftliche Beratung, Ausbildung für Vermarktung und Kredit, Helvetas-Projekt); Unterstützung und Ausbildung für Bauernfamilien, Bauernverbände und Verarbeitungsbetriebe im Rahmen des Privatisierungsprozesses in der Landwirtschaft (Caritas).

\section{Russland}

Die zweite Jahrestagung des Bundes über die Zusammenarbeit in Osteuropa und in der GUS fand am 17. Juni 1997 in Bern statt (2. Focus Osteuropa). Nach Rumänien im Jahre 1996 war das von der Konferenz 1997 untersuchte Land die Russische Föderation. Der Gesamtbetrag der Verpflichtungen der Schweiz für Projekte in Russland belief sich Ende 1996 auf 193 Millionen Franken (davon 100 Millionen Franken für Kreditgarantien, 57 Millionen Franken für Finanzhilfe und 36 Millionen Franken für technische Zusammenarbeit). Dies mag in einem so riesigen Land wie ein kleiner Tropfen erscheinen. So konzentriert die Schweiz ihre Mittel auf bestimmte Sektoren und Regionen. Russland ist ein Land, das reich an Rohstoffen sowie an qualifizierten Arbeitskräften und Wissenschaftlern ist. Daher liegt die Priorität nicht bei der Grundausbildung oder dem Spitalbau, sondern die Hauptstossrichtungen der schweizerischen Zusammenarbeit sind folgende:

๑ Unterstützung der Demokratisierung und der Wahrung der Menschenrechte;

口 Nukleare Sicherheit;

๑ Umweltschutz, Erhaltung der biologischen Vielfalt;

- Förderung kleiner und mittlerer Unternehmen in drei Pilotregionen (Woronesch, Kaluga und Nischni Novgorod), um den Zugang der KMU zu Knowhow und Krediten zu erleichtern.

8. Für Informationen über die schweizerische Zusammenarbeit in Kirgistan verweisen wir auf den DAC-Bericht über die Prüfung des Programms der Entwicklungszusammenarbeit der Schweiz, auf die Helvetas-Zeitschrift Partner Nr. 148, Mai 1997, sowie auf die Wirtschaftszeitschrift des Schweizerischen Bankvereins, Der Monat in Wirtschaft und Finanz, Mai 1997.

9. Siehe Pressedossier zur 2. Jahrestagung des Bundes über die Zusammenarbeit in Osteuropa und in der GUS, «Nouveau départ pour l'Europe de l’Est. Spécial Russie. Que fait la Suisse?», Bern, 17. Juni 1997. 


\section{$\square$ Prioritäre Bereiche der schweizerischen Osteuropa-Zusammenarbeit}

Die wichtigsten durch die technische Zusammenarbeit unterstützten Bereiche sind folgende: Gesundheit/Soziales, Wissenschaft/Kultur, Wirtschaft/Ausbildung (diese drei Bereiche mobilisieren 57\% der Verpflichtungen), Landwirtschaft, Politik und Staatsaufbau, Energie/Umwelt. Die finanzielle Zusammenarbeit wendet sich vor allem folgenden Sektoren zu: Energie (31\% der Hilfe), Umwelt (22\%), Gesundheitswesen (20\%) und Infrastruktur (13\%), diverse Bereiche (14\%).

\section{Gesundheitswesen}

Das in den letzten Jahren errichtete Netz an Spitalpartenschaften ist eine interessante Form der Zusammenarbeit, welche Personen, Hilfswerke, Spitäler, rund hundert Schweizer Gemeinden und Unternehmen umfasst. Die DEZA unterstützt 17 Partnerschaften zwischen Spitälern in der Schweiz (in verschiedenen Kantonen) und in Osteuropa. Diese Partnerschaften konzentrierten sich anfänglich auf Tschechien, Ungarn und Polen und verlagerten sich anschliessend vor allem nach Albanien, Bulgarien und Rumänien. Die Spitalpartnerschaften werden von der Vereinigung $\mathrm{H}+$ (früher: Vereinigung Schweizerischer Krankenhäuser VESKA) verwaltet und von der DEZA finanziell unterstützt. Hauptziele sind die Verbesserung der Hygiene, die Errichtung sanitärer Anlagen oder die Maschinenwartung ${ }^{10}$. Die Vereinigung $\mathrm{H}+$ verwaltet auch ein Ausbildungsprogramm für Spitalmanagement. Weitere Gesundheitsprogramme werden von der DEZA gefördert: Unterstützung des Universitätsspitals und des nationalen Gesundheitsinstituts in Tirana (Albanien), Caritas-Gesundheitsprogramm in Albanien. 


\section{QUELLEN}

Bundesrat, Botschaft zum allgemeinverbindlichen Bundesbeschluss über die Zusammenarbeit mit den Staaten Osteuropas, vom 19. September 1994, Bern, Bundesblatt 1994, Band V, S. 553 - 580 (Botschaft Nr. 94.083).

Bericht zur Aussenwirtschaftspolitik 96/1 + 2 sowie Botschaften zu Wirtschaftsvereinbarungen, vom 15. Januar 1997, Botschaft Nr. 97.002.

Botschaft über die Beteiligung der Schweiz an der Kapitalerhöhung der Europäischen Bank für Wiederaufbau und Entwicklung (EBWE), vom 13. November 1996, Botschaft Nr. 96.089.

OECD, Série d'examens en matière de coopération pour le développement No 20, Suisse, Paris, Comité d'aide au développement (CAD), 1997.

DEZA, BAWI, Jahresbericht 1996, Zusammenarbeit mit Osteuropa, Bern, 1997, 16 S.

Die Schweiz, Osteuropa und die GUS, Eine Dokumentation zum Unterstïtzungsprogramm des Bundes für die osteuropäischen Staaten und die Staaten der GUS, Bern, DEZA und BAWI, 4. überarbeitete Auflage, 1997/1998, 30 S.

DEZA, Eine Dokumentation über die Projekte der technischen Zusammenarbeit mit den osteuropäischen Staaten, 2. Rahmenkredit, Bern, DEZA, Januar 1998, 103 S. Liste der Projekte technischer Zusammenarbeit.

Pressedossier betreffend die zweite Jahrestagung des Bundes über die Zusammenarbeit in Osteuropa und in der GUS «Nouveau départ pour l'Europe de l'Est. Spécial Russie. Que fait la Suisse ?», Bern, 17. Juni 1997.

Weltbank, Weltentwicklungsbericht 1996, von der Planwirtschaft zur Marktwirtschaft, Washington, Weltbank, 1996.

Mosquito, «Osthilfe: Zankapfel zwischen den politischen Fronten, Osthilfe der DEZA und des BAWI, Hilfswerke in Osteuropa und Zentralasien », Mosquito Nr. 2., März 1997.

Agefi, Quotidien des affaires et de la finance, 15. April 1997 «BERD»; 16. April 1997 «L'assemblée annuelle de la BERD souligne la fragilité du processus de transition »; 18. Juni 1997 «Conférence annuelle sur la coopération avec l'Europe de l'Est».

Le Mois économique et financier, (Der Monat in Wirtschaft und Finanz), Schweizerischer Bankverein, Januar/Februar 1997 «Vaste soutien aux pays en transition»; Mai 1997 «Asie centrale. La coopération entre la Suisse et les républiques centre-asiatiques».

Neue Zürcher Zeitung, 4. April 1997 «Schwierige Landreform in Litauen. Transfer von Know-how mit Schweizer Hilfe»; 15. April 1997 «EBRD-Rückzug aus erfolgreichen Reformländern»; 26./27. April 1997 «Einigung über die Absicherung des Tschernobyl-Sarkophags»; 30. April 1997 «Osteuropabank-Unterstützung im Nationalrat unbestritten»; 23. Juni 1997 «Ein Investitionsland von steigender Attraktivität. Bawi und Deza zur Schweizer Präsenz in Russland»; 28./29. Juni 1997 «Rücktritt des EBRD-Präsidenten»; 22./23. November 1997 «Weitere Mittel für Tschernobyl». Partner, Dossier: Kirghistan: un pays en transition, Helvetas, Partner Nr. 148, Mai 1997.

La Vie économique, revue de politique économique (Die Volkswirtschaft, das Magazin für Wirtschaftspolitik), 2/1997 «Développements économiques en Europe centrale et orientale: Présence de la Suisse».

INTERNET-ADRESSE:

DEZA: http://www. sdc-gov. ch

Europäische Bank für Wiederaufbau und Entwicklung: http://www. ebrd. com 